\title{
Renormalization group treatment of two interacting magnetic impurities in a metal
}

\author{
J.W. Rasul \\ Physics Department, University of Michigan, Ann Arbor, MI, USA \\ P. Schlottmann \\ Department of Physics and Center for Materials Research and Technology, Florida State University, \\ Tallahassee, FL, USA
}

\begin{abstract}
We consider two resonant level impurities, each of which hybridizes with, and has an attractive interaction with, an electron band (in such a way as to mimic the Kondo effect). They interact with each other via a hopping matrix element and an interaction between the localized fermions (so as to resemble the RKKY interaction between the impurities). We obtain the momentum space RG equations for the model, which are discussed in terms of Ward cancellations. Expressions for the static and dynamic susceptibilities in uniform and staggered fields are obtained. No anomalies are found, probably as a result of the broken spin-rotational invariance of the model.
\end{abstract}

The two-impurity Kondo problem has played a central role in the field of correlated electron physics - at a heuristic level the competition between RKKY and Kondo compensation of the local moments is crucial for an understanding of the existence of heavy fermion and valence fluctuation behaviour. Recently this problem has undergone a resurgence in the light of numerical RG treatments and conformal invariance techniques [1] that show the existence of a nontrivial fixed point separating the extremes of behaviour corresponding to the Kondo and antiferromagnetic fixed points. Associated with this fixed point are a diverging specific heat and staggered susceptibility, although the uniform susceptibility remains finite.

If the physical results associated with these treatments are to have consequences for the lattice problem then it is essential that a more conventional many-body scheme be found that is capable of reproducing these results. To this end we have studied a two impurity extension of the resonant level model [2], which so successfully reproduced the low and intermediate temperature properties of the single-ion Kondo model.

In broad terms, the steps behind the derivation of this model are; (1) the representation of spin operators by fermion operators $\left(S^{+} \rightarrow d^{+}, S^{-} \rightarrow d\right.$,

Correspondence to: J.W. Rasul, Physics Department, University of Michigan, Ann Arbor, MI 48109, USA.
$S^{z} \rightarrow d^{+} d-1 / 2$ ) (2) bosonization of the conduction electron states, an extraction of the electron spin density operators, and (3) a reintroduction of spinless fermion operators $\left(c_{k}\right)$ to represent these. The result of all these rearrangements is that the spin flip terms become replaced by an effective hybridization between the local and band states, while the longitudinal terms result in a two-body interaction between the local electron and band electron densities.

The main point of this model is the Ward cancellation between self energy and vertex parts, so that the effective two-body interaction stays small and a set of Fermi liquid relations can be derived for the lowtemperature regime. To generalize this model to the case of two impurities, however, it is necessary to build in the RKKY interaction externally, since those processes that mediate the RKKY interaction are actually obscured by the bosonization of the electron states. We therefore follow the now standard procedure of adding an additional term to the Hamiltonian $[1,3]$, which when written in terms of the $d$ operators, takes on the form of a hopping between the two sites together with an interaction between the charge densities at the two sites.

The final step in writing the model consists of introducing electron states that have even and odd parity with respect to the midpoint of the impurities. The same is done for the $d$ states which then represent singlet or triplet impurity combinations. The Hamilto- 
nian then reads

$$
\begin{aligned}
H= & \sum_{k} \varepsilon_{k} c_{k, p}^{+} c_{k, p}+\varepsilon \sum_{p}\left(d_{p}^{+} d_{p}-\frac{1}{2}\right) \\
& -U \sum_{p}\left(d_{p}^{+} d_{p}-\frac{1}{2}\right) \sum_{k, k_{1}, p_{1}}\left[1+p_{1} A\right] c_{k, p_{1}}^{+} c_{k_{1}, p_{1}} \\
& +\mathrm{i} U B\left(d_{+}^{+} d_{-}+d_{-}^{+} d_{+}\right) \sum_{k, k_{1}}\left[c_{k,+}^{+} c_{k_{1},-}-c_{k,-}^{+} c_{k_{1},+}\right] \\
& +\sum_{k, p}\left[V_{+}\left(c_{k,+}^{+} d_{+}+\text {h.c. }\right)+\mathrm{i} V_{-}\left(c_{k,-}^{+} d_{-}+\text {h.c. }\right)\right] \\
& +t\left(d_{+}^{+} d_{+}-d_{-}^{+} d_{-}\right)+G d_{+}^{+} d_{+} d_{-}^{+} d_{-}
\end{aligned}
$$

where $p=+,-$ denotes the parity, while $A, B, V_{-}$and $V_{+}$depend on $k_{\mathrm{F}}$ and the inter-impurity separation. We proceed to study the renormalization of $t$ and $V$ by considering perturbation theory in $U$. The first step is to define the vertex function $\Gamma_{p, p_{1}}(\omega)$ associated with the interaction $U$ such that it reduces to its bare value

$\Gamma_{p, p_{1}}(\omega=D)=(1+p A) \delta_{p, p_{1}}-p(-1)^{\prime} B \delta_{p,-p_{1}}$

at high energies.

Proceeding to the calculation of diagrammatic corrections to $\Gamma$, it is found that to second order in $U$ the logarithmic terms cancel but that the next leading logarithms (at order $U^{3}$ ) do not. However, when combined with the self-energy correction to the delectron weight $d(\omega)$, it is found that these nextleading order divergences cancel, i.e. the derivative of $\Gamma(\omega) d(\omega)$ with respect to $\omega$ is zero. The effective interaction therefore remains at its bare value under reductions in the bandwidth, in a similar way as for the X-ray edge problem [4] and the TomonagaLuttinger model [5].

Scaling does however affect both the hybridization width $\Omega^{ \pm}=\pi \rho\left|V_{ \pm}\right|^{2} d(\omega)$ and the hopping matrix element $t$. Figure 1 shows the effects of renormalizing the latter, which leads to a correction

$\delta t=-4(U \rho)^{2} \int_{\omega}^{D} \mathrm{~d} \omega_{1}\left|d\left(\omega_{1}\right) \Gamma_{+,-}\right|^{2} t\left(\omega_{1}\right) / \omega_{1}$

which after integrating, and using the fact that $\Gamma$ stays at its bare value, yields $t(\omega)=t[\omega / D]^{4 B^{2} U^{2} \rho^{2}}$. Implicit in this expression is the assumption that the impurity states are long-lived. The hopping itself or the hybridization provide a cutoff at a finite time (infrared cutoff), which is itself to be determined self-consistently. This problem is closely related to the electronassisted tunneling of an atom [6]. Here the diffusion of the atom is slowed down by electron-hole excitations it creates while moving. The self-consistent treatment of the hopping leads then to a delocalization of the atom.

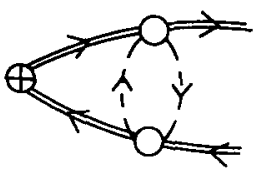

(a)

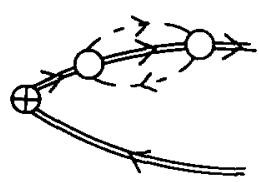

(b)

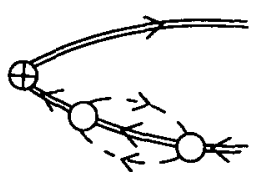

(c)

Fig. 1. Renormalization of the hopping matrix element (denoted by an encircled plus-sign). Dashed lines represent band electron propagators, while the double solid line represents a dressed impurity propagator. The empty circles denote the vertex function discussed in the text. Diagram (a) represents renormalization of the hopping vertex while (b) and (c) are self-energy corrections.

Applying the same approach to the hybridization (fig. 2) we obtain an integral equation

$V_{p}\left(\omega^{\prime}\right)=V_{p}-U \rho \int_{\omega^{\prime}}^{D} \mathrm{~d} \omega d(\omega) \sum_{p_{1}} \Gamma_{p, p_{1}}(\omega) V_{p_{1}}(\omega) / \omega$

which leads to a different scaling exponent, namely $V(\omega)=V[\omega / D]^{\lambda}$ where $\lambda=U \rho\left[1-\sqrt{A^{2}+B^{2}}\right]$. Thus both the hybridization (characterizing the Kondo resonance width) and the hopping (characterizing the RKKY spin flip processes) scale, but with different exponents. If self-consistency is imposed then these quantities depend on a more general set of parameters, including $\varepsilon$ and $t$. It is found that the resonance width and hopping rate increase monotonically with $\omega$ and $T$.

We next proceed to study the homogeneous and staggered field susceptibilities

$$
\begin{aligned}
& \chi(z)=-\left\langle\left(d_{1}^{+} d_{1}+d_{2}^{+} d_{2}\right) ;\left(d_{1}^{+} d_{1}+d_{2}^{+} d_{2}\right)\right\rangle_{2}, \\
& \chi_{s}(z)=-\left\langle\left(d_{1}^{+} d_{1}-d_{2}^{+} d_{2}\right) ;\left(d_{1}^{+} d_{1}-d_{2}^{+} d_{2}\right)\right\rangle_{z},
\end{aligned}
$$

which, because of the invariance of the effective interaction under scaling, together with its overall weak-

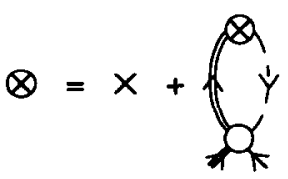

Fig. 2. Integral equation for the hybridization (denoted by an encircled cross). The uncircled cross denotes the bare hybridization. 
ness, can be factorized into a product of local electron Greens functions that depend only on the renormalized hybridization width and hopping rate. The resulting expressions are easy to evaluate and at low temperatures $\left(\chi_{ \pm}(z)=-\left\langle\left\langle d_{ \pm}^{+} d_{ \pm} ; d_{ \pm}^{+} d_{ \pm}\right\rangle_{z}\right)\right.$ we find that

$$
\begin{aligned}
\chi_{ \pm}(0)= & (1 / \pi) \Omega_{0}^{ \pm} /\left(\left(\Omega_{0}^{ \pm}\right)^{2}+\left(\varepsilon \pm t_{0}\right)^{2}\right), \\
\chi_{s}(0)= & \operatorname{Re}\left(\Omega_{0}^{+}-\Omega_{0}^{-}+2 \mathrm{i} t_{0}\right)^{-1} \\
& \times \ln \left[\frac{\left(\Omega_{0}^{+}+\mathrm{i}\left(\varepsilon+t_{0}\right)\right)}{\left(\Omega_{0}^{-}+\mathrm{i}\left(\varepsilon-t_{0}\right)\right)}\right] / \pi,
\end{aligned}
$$

where $t_{0}, \Omega_{0}^{ \pm}$denote the zero-temperature, zerofrequency limit of these quantities. Both of these susceptibilities have a maximum when $\varepsilon=t_{0}=0$, i.e. the impurity triplet and singlet states are resonant with the Fermi level, in which case (for $\Omega_{0}^{-}$tending to zero) we have

$\chi_{-}(0)=1 /\left(\pi \Omega_{0}^{-}\right), \quad \chi_{s}=\ln \left(\Omega_{0}^{+} / \Omega_{0}^{-}\right) /\left(\pi \Omega_{0}^{+}\right)$.

These quantities would diverge in the limit of vanishing effective hybridization, since in this case one of the resonance widths vanishes while the other stays finite. In this context we note that a similar result was obtained in a $1 / N$ calculation [3] if one of the resonance widths was tuned so as to vanish. In general, however, these response functions do not diverge, which is probably a result of the rotational invariance that is broken by representation of the spin operators in terms of fermions and the subsequent expansion in $U$.

At high temperatures the resonant widths can again be neglected, and the susceptibilities reduce to their expected Curie-Weiss behaviour with an antiferromagnetic Weiss temperature of the order of $\Omega_{0}^{ \pm}$. We have also studied the dynamic response functions and find at high temperatures a Lorentzian behaviour in the absorbtion spectra. At low temperatures a twopeak structure is obtained, with peaks at $\varepsilon \pm t_{0}$. These energies correspond essentially to spin flips of the local states. The typical behaviour of $\chi_{ \pm}^{\prime \prime}(\omega) / \omega$ is shown in fig. 3. Finally, we have examined the effects of $G$, the longitudinal RKKY component, perturbatively. We find that, within an RPA scheme, both staggered and uniform responses are enhanced, also the staggered susceptibility is enhanced further.

In summary, we have examined a two-impurity

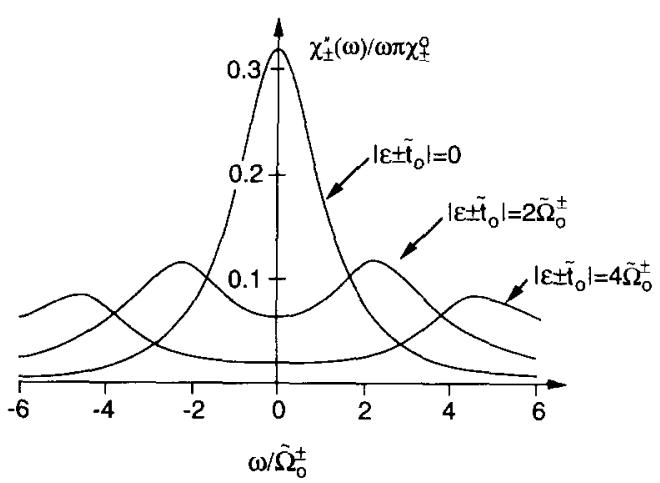

Fig. 3. Dynamical susceptibility at zero temperature versus frequency for three values of the d-level energy. As this energy increases from zero the quasielastic peak is suppressed and thresholds appear at higher energy.

generalization of the resonant level model, including an RKKY coupling between the spins. We find that the Ward cancellation leads to the effective interaction being small, and that as a result the dynamically generated hybridization and RKKY spin flip are frequency dependent, with different power law exponents. The homogenous and staggered field susceptibilities do not diverge except for specially tuned values of the parameters, rather like the model treated in ref. [3]. We attribute the lack of critical behaviour to the breaking of spin rotational invariance.

\section{References}

[1] B.A. Jones, C.M. Varma and J.W. Wilkins, Phys. Rev. Lett. 61 (1988) 1415; A. Ludwig and I. Affleck, Phys. Rev. Lett. 61 (1991) 161.

[2] P. Schlottmann, J. Magn. Magn. Mater. 7 (1978) 72; P. Schlottmann, J. Phys. (Paris) Colloq. 39 (1978) C6-1486; P.B. Vigman and A.M. Finkelstein, Sov. Phys. JETP 48 (1978) 102; P. Schlottmann, Phys. Rev. B 25 (1982) 4815.

[3] B.A. Jones, B.G. Kotliar and A.J. Millis, Phys. Rev. B 39 (1989) 3415.

[4] P. Nozieres and C.T. de Dominicis, Phys. Rev. B 178 (1969) 1097.

[5] S. Tomonaga, Prog. Theor. Phys. 5 (1950) 544; J.M. Luttinger, J, Math. Phys. 4 (1963) 1154.

[6] K. Yamada, A. Sakurai and M. Takeshige, Prog. Theor. Phys. 70 (1983) 73; K. Vladar and A. Zawadowski, Phys. Rev. B 28 (1983) 1564, 1582, 1596; J.L. Black, K. Vladar and A. Zawadowski, Phys. Rev. Lett. 56 (1982) 286. 